\title{
Evaluation of Enamel Surface after Removal of Adhesive Resin: An in Vitro Study
}

\author{
Dr Geeta Pyakurel,' Prof Dr Gazi Shamim Hassan,2 Asso Prof Dr Mahmood Sajedeen³ \\ 'Orthodontist, 2Professor, ${ }^{3}$ Asso Professor, Dept of Orthodontics, \\ Bangabandhu Sheikh Mujib Medical University, Bangladesh
}

Correspondence: Dr Geeta Pyakurel; Email: geetapyakureh1@gmail.com

\section{ABSTRACT}

Introduction: Preservation of the enamel surface during the removal of orthodontic appliance is an essential aspect for clinicians. However, various therapeutic measures can affect the enamel surface. The objective of the research is to evaluate the roughness of enamel surface after the removal of adhesive resin.

Materials \& Method: 40 extracted human premolar teeth were randomly divided into two groups and the enamel surfaces were initially subjected to profilometer for the assessment of surface roughness. Following bracket bonding, debonding was done and adhesive resin was removed by stainless steel bur in Group A and tungsten carbide bur in Group B. Again, the surface roughness was measured by profilometer. Independent t-test was performed to compare the enamel surface roughness between two groups and paired t-test to compare the enamel surface roughness within the groups.

Result: The mean average surface roughness of stainless steel bur was $27.009 \pm 4.8420 \mu \mathrm{m}$ and tungsten carbide was $31.426 \pm$ $5.0956 \mu \mathrm{m}$. The result showed that there was significant difference in enamel surface roughness between two groups.

Conclusion: The roughness values were found to be significantly decreased with the use of stainless steel bur (SS) than tungsten carbide bur (TC).

Keywords: Bracket debonding, Enamel surface roughness, Profilometer, Stainless steel bur, Tungsten carbide bur.

\section{INTRODUCTION}

Orthodontic treatment plays a vital role in enhancing esthetics, functions and self-esteem in a patient. The bonding of brackets to tooth surface is temporary as the brackets and adhesive need to be removed after the completion of active orthodontic treatment.' Debonding aims to remove the orthodontic attachments and the remaining adhesive from the tooth surface and to restore the surface to its pretreatment state. $^{2}$

Residual adhesive resin on the tooth surface after debonding results enamel roughness that cause discoloration of tooth, plaque accumulation and enamel decalcification. ${ }^{3}$ If there is loss of enamel structure during debonding and cleaning it will develop tooth sensitivity. The amount of enamel loss during bonding and debonding is 5-150 micro meters. ${ }^{4}$

One of the goals of an Orthodontist is to restore the enamel surface as closely as possible to its pretreatment condition without inducing iatrogenic damage at the termination of fixed orthodontic treatment. ${ }^{5}$ Different methods used in the previous studies for the removal of adhesive resin from the enamel surface after bracket debonding are: tungsten carbide bur at high speed followed by polishing with pumice, enhance rubber points and finally with brown and green cups. ${ }^{6}$ Other methods of removing adhesive resin are: fiber reinforced composite bur, ${ }^{7}$ scaler, ${ }^{8}$ intra-oral sandblasting ${ }^{9}$ and low level carbondioxide laser. ${ }^{10}$

The enamel surface must be smoothened and polished to prevent the plaque accumulation..$^{11,12}$ The research for the best technique that would leave smooth and ideal finish for the tooth surface without loss of tooth structures is still ongoing. So, this study was conducted to evaluate the roughness of enamel surface after bracket debonding and adhesive resin removed by stainless steel and tungsten carbide bur.

\section{MATERIALS AND METHOD}

In vitro experimental study was performed on 40 extracted human premolar teeth in Department of 
Orthodontics, BSMMU, Dhaka and Institute of Fuel Research and Development, BCSIR, Dhaka. The collected teeth were extracted for the orthodontic purpose. The study was conducted after receiving ethical approval from Institutional Review Board of Bangabandhu Sheikh Mujib Medical University. Simple random sampling method was used and the sample size was calculated by using the following formula:

$$
N=\frac{\left(Z_{a}+Z_{\beta}\right)^{2} \times\left(\sigma_{1}{ }^{2}+\sigma_{2}{ }^{2}\right)}{\left(\mu_{1}-\mu_{2}\right)^{2}}
$$

Extracted both maxillary and mandibular premolars with intact buccal surface were included in the study where as teeth with caries or restorations, visible cracks and enamel defects like hypocalcifications or fluorosis were excluded. 40 human premolar teeth were collected from the stored vessel in the normal saline and were divided into two groups; (Group A and Group B) 20 in each group.

The roots of all the teeth were cut off approximately $2 \mathrm{~mm}$ below the cemento-enamel junction. The crown was individually embedded horizontally in autopolymerizing acrylic resin block with at least $2 \mathrm{~mm}$ of buccal enamel exposure. Each block was of same size $(25 \times 25 \times 20) \mathrm{mm}^{3}$. The blocks were kept in distilled water at room temperature during the time of experiment. Before bonding, the surface profile of each tooth was measured using profilometer (figure 1) with a stylus tip oriented perpendicular to the enamel surface during scanning. The measurements were recorded in micrometer using computer. The measurement parameters were as follows:

1. Ra (Arithmetic mean value of surface roughness): Ra indicates average roughness.

2. $\mathrm{Rq}$ (Root mean square roughness): Indicates root mean square deviation.

3. Rt (Maximum roughness height): Indicates

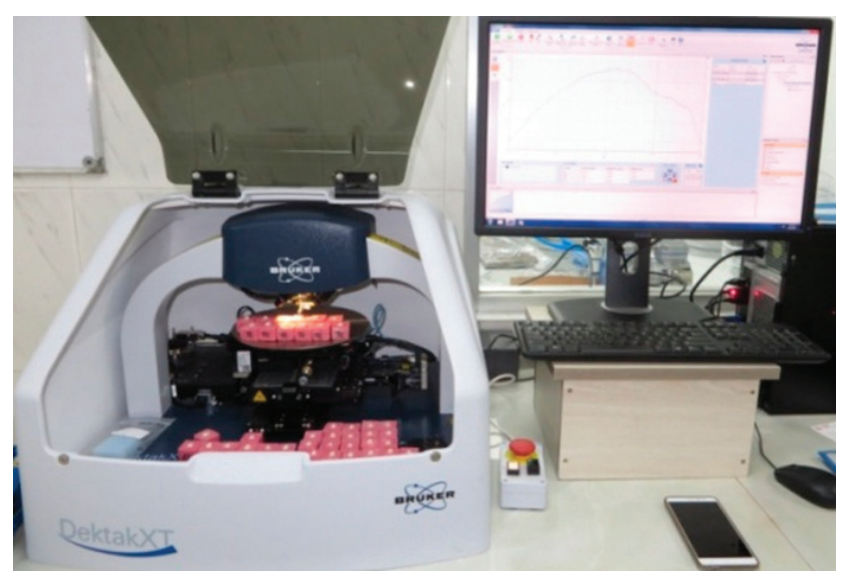

Figure 1: Profilometer maximum peak-to valley height over the sampling length.

4. Rz (Mean roughness depth): Indicates mean vertical distance between the highest peak and the deepest valley.

After the measurement of first surface roughness, buccal surfaces of all sample teeth were cleansed with fluoride free pumice (Prolax, India) and water. Each tooth was etched with $37 \%$ phosphoric acid semi gel (Meta biomed co.Itd.) for 15 seconds, rinsed thoroughly with water/air spray combination for 10 seconds and dried with oil-free compressed air until a frosty white etched area was observed. Ortho solo primer (Ormco Corp.) was applied to the etched surface of enamel and Archist ${ }^{R} 0.018$ slot Roth, premolar metal bracket was bonded with Enlight (Ormco Corp.) to the prepared crown. Pressure was applied and excess material around the bracket base was removed with the tip of an explorer and cured with a LED light curing unit (D-Lux, DiaDent, Korea) for 10 seconds per tooth according to manufacturer's instructions. The specimens were stored in distilled water at room temperature for 24 hours and debonded with a straight bracket debonding plier (Ormco, Corp.) holding against the wings of the bracket and squeezed. This method has been described as the safest way to remove the metal brackets. ${ }^{13,14}$ Both the bonding and debonding protocol were same for all 40 teeth.

Then, the remaining adhesive resin on the enamel surface was removed with stainless steel (SS) (Alston, England) bur at low speed hand-piece in Group A and tungsten carbide (TC) bur (30 fluted, Germany) at low speed hand-piece in Group B. Great care was taken not to damage the enamel. All the bonding, debonding and adhesive removal procedures were performed by the same operator. A new bur was used for each tooth. Complete removal of adhesive remnants were ensured clinically by visual inspection under a dental operating light. After the removal of adhesive resin, specimens were subjected to profilometer for the second time and the roughness values were recorded.

\section{RESULT}

The surface roughness of enamel was measured by profilometer in $\mu \mathrm{m}$ for 20 samples in each experimental group. The results of surface roughness for both SS and TC groups, before and after the treatment were summarized in (Table 1). Mean and standard deviation for all the roughness parameters (Ra, Rq, Rt, Rz) were calculated two times, before treatment and after resin removed with SS and TC bur following 
Table 1: Comparison of enamel surface roughness before and after treatment

\begin{tabular}{|c|c|c|c|c|}
\hline Parameters & Groups & Number & Mean \pm SD & $p$-Value* \\
\hline \multirow{4}{*}{$\begin{array}{l}\text { Ra }(\mu \mathrm{m}) \\
\text { (Average roughness) }\end{array}$} & Pre-treated SS Ra & \multirow{2}{*}{20} & $21.966 \pm 4.5204$ & \multirow{2}{*}{$0.000^{*}$} \\
\hline & Treated SS Ra & & $27.009 \pm 4.8420$ & \\
\hline & Pre-treated TC Ra & \multirow{2}{*}{20} & $21.966 \pm 3.3024$ & \multirow{2}{*}{$0.000^{*}$} \\
\hline & Treated TC Ra & & $31.426 \pm 5.0956$ & \\
\hline \multirow{4}{*}{$\begin{array}{l}\mathrm{Rq}(\mu \mathrm{m}) \\
\text { (Root mean } \\
\text { square roughness) }\end{array}$} & Pre-treated SS Rq & \multirow{2}{*}{20} & $26.761 \pm 6.2871$ & \multirow{2}{*}{0.018} \\
\hline & Treated SS Rq & & $32.912 \pm 9.2515$ & \\
\hline & Pre-treated TC Rq & \multirow{2}{*}{20} & $26.760 \pm 4.2255$ & \multirow{2}{*}{$0.000^{*}$} \\
\hline & Treated TC Rq & & $39.136 \pm 8.2678$ & \\
\hline \multirow{4}{*}{$\begin{array}{l}\text { Rt }(\mu \mathrm{m}) \\
\text { (Maximum roughness } \\
\text { height) }\end{array}$} & Pre-treated SS R† & \multirow{2}{*}{20} & $29.889 \pm 6.8485$ & \multirow{2}{*}{$0.000^{*}$} \\
\hline & Treated SS Rt & & $36.467 \pm 5.6370$ & \\
\hline & Pre-treated TC Rt & \multirow{2}{*}{20} & $29.889 \pm 4.3468$ & \multirow{2}{*}{$0.000^{*}$} \\
\hline & Treated TC R† & & $41.977 \pm 7.8174$ & \\
\hline \multirow{4}{*}{$\begin{array}{l}\text { Rz }(\mu \mathrm{m}) \\
\text { (Mean roughness depth) }\end{array}$} & Pre-treated SS Rz & \multirow{2}{*}{20} & $35.557 \pm 7.7195$ & \multirow{2}{*}{0.000} \\
\hline & Treated SS Rz & & $62.493 \pm 1.1279$ & \\
\hline & Pre-treated TC Rz & \multirow{2}{*}{20} & $35.558 \pm 1.1488$ & \multirow{2}{*}{$0.000^{*}$} \\
\hline & Treated TC Rz & & 73. $777 \pm 1.6380$ & \\
\hline
\end{tabular}

SS = Stainless steel, TC= Tungsten carbide

*= Statistically significant at $p<0.05$

debonding. At first the normality of the data were checked by Shapiro-Wilk and Kolmogorov-Smirnov test and parametric test was performed. The mean $\mathrm{Ra}, \mathrm{Rq}, \mathrm{R} \dagger$ and Rz values were $(21.966 \pm 4.5204,26.761$ \pm 6.2871, $29.889 \pm 6.8485,35.557 \pm 7.7195) \mu \mathrm{m}$ before and $(27.009 \pm 4.8420,32.912 \pm 9.2515,36.467 \pm 5.6370$, $62.493 \pm 1.1279) \mu \mathrm{m}$ respectively, after resin removal with stainless steel bur. The mean Ra, Rq, Rt and Rz values for tungsten carbide bur were (21.966 \pm 3.3024 , $26.760 \pm 4.2255,29.889 \pm 4.3468,35.558 \pm 1.1488) \mu \mathrm{m}$, before and $(31.426 \pm 5.0956,39.136 \pm 8.2678,41.977$ $\pm 7.8174,73.777 \pm 1.6380) \mu \mathrm{m}$ respectively, after resin removal. Paired t-test showed a statistically significant difference in surface roughness within the groups for both SS and TC bur, $(p<0.05)$. Mean roughness values were increased after the resin removed from the teeth in both methods when compared with the roughness values of intact tooth. Independent t-test indicated a statistically significant difference between two groups for all the roughness parameters after the removal of adhesive resin $(p<0.05)$.

\section{DISCUSSION}

The main concern of an orthodontist after debonding is to re-establish the original smoothness of enamel surface. Debonding procedure and removal of adhesive resin from the tooth surface may damage the external layer of the enamel which presents higher mineral and fluoride content than do the deeper layers. ${ }^{715}$ The loss of surface enamel makes it prone to decalcification. ${ }^{16}$

In this in vitro study, the mean values for all the roughness parameters were increased after the removal of resin in both the methods. Paired T-test showed that there was a statistically significant difference within the groups before and after the removal of resin. This finding is in agreement with previous studies. $6,7,11,12$ Independent t-test showed a statistically significant difference between two groups after the removal of resin. Mean roughness values after the removal of resin were greater in TC group than SS group (Graph 1). This finding was different to previous research.5,17 The difference in effect of SS and TC bur between these studies and current study may be due to several variables like adhesive resin and bonding used, debonding technique, speed of rotatory instrument and methods used in assessment of surface roughness. ${ }^{12}$ Previous studies have used scanning electron microscope (SEM) for the assessment of enamel surface roughness. In SEM, surface roughness of specimens cannot be quantitatively evaluated and the obtained results are completely subjective. ${ }^{18}$ In the current study, profilometer was used for quantitative 


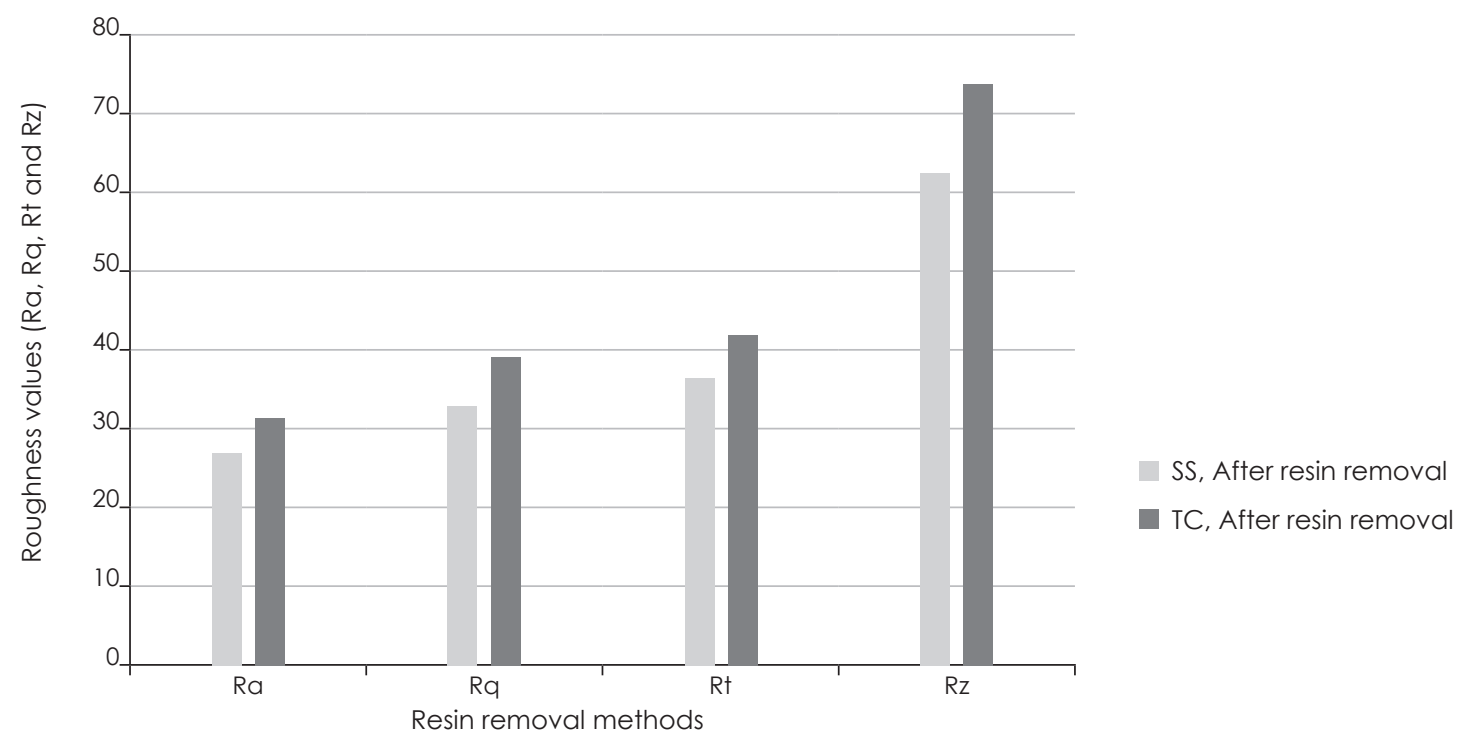

Graph 1: Showing Ra, Rq, Rt, Rz values after resin removal with SS and TC bur.

assessment of enamel surface. This method has been used in recent studies. ${ }^{9,12,19}$

we focused on standardizing and controlling all variables and only investigated two types of burs which were used under the same conditions. In order to maximize accuracy, a new bur was used for each tooth. The data of this study showed the mean of average surface roughness after resin removal with SS group was $27.009 \mu \mathrm{m}$ which was less than TC group. Although the resin removal procedures caused deterioration of the enamel surface, no clinical problem was observed because the thickness of normal enamel is from 1000$2000 \mu \mathrm{m}$ which contained a fluoride-rich layer of 50 $\mu \mathrm{m}$ depth..$^{20}$ So SS bur can be used in clinical practice for the removal of adhesive resin after orthodontic debonding. This study was an in vitro study so there was potential limitation of studying an in vivo phenomenon out of its natural setting. The completion of the cleaning stage was only determined with visual examination of the enamel surface. Different clinicians can have different finishing goals for this stage.

\section{CONCLUSION}

Both the burs used in the current study for the removal of adhesive resin from the enamel surface of teeth during debonding caused some damage to the enamel surface. The roughness values were found to be significantly decreased with the use of stainless steel bur than tungsten carbide bur. Stainless steel bur at low speed produced a smooth enamel surface and was able to preserve the tooth surface closer to its original state.

\section{Acknowledgement}

Sincere thanks to Prof. Dr. Rabindra Man Shrestha, Department of Orthodontics, Kantipur Dental College, Kathmandu, Nepal 


\section{REFERENCES}

1. Bishara SE, VonWald L, Laffoon JF, Warren J. Effect of self- etch primer/adhesive on the shear bond strength of orthodontic brackets. Am J Orthod Dentofacial Orthop. 2001; 119:621-624.

2. Arhun N, Arman A. Effects of orthodontic mechanics on tooth enamel. Semin Orthod. 2007; 13(4):281-291.

3. Shamsi AH Al, Cunningham J Leo, Lamey PJ, Lynch E. Three-dimensional measurement of residual adhesive and enamel loss on tooth after debonding of orthodontic brackets: An in-vitro study. Am J Orthod Dentofacial Orthop. $2007 ; 131$ (301):9-15.

4. Krell KV, Courey JM, Bishara SE, 1993. Orthodontic bracket removal using conventional and ultrasonic debonding techniques, enamel loss and time requirements. Am J Orthod Dentofacial Orthop. 1993; 103:258-266.

5. Retief D H, Denys F R. Finishing of enamel surfaces after debonding of orthodontic attachments. Angle Orthod. 1979; $49(1): 1-10$.

6. Campbell P M. Enamel surfaces after orthodontic bracket debonding. Angle Orthod. 1995; 65 (2):103-110.

7. Karan S, Kircelli B H, Tasdelen B, 2010. Enamel surface roughness after debonding. Angle Orthod. 2010; 80(6):1081-1088.

8. Rouleau B D, Marshall G W, Cooley Robert O. Enamel surface evaluations after clinical treatment and removal of orthodontic brackets. Am J Orthod Dentofacial Orthop. 1982May; p.423-426.

9. Kim S S, Park W K, Son W S, Ahn H S, Ro J H, Kim Y Ds. Enamel surface evaluation after removal of orthodontic composite remnants by intraoral sandblasting: A 3-dimensional surface profilometry study. American Journal of Orthodontics and Dentofacial Orthopedics. 2007; 132(1):71-6.

10. Obata A, Tsumura T, Niwa K, Ashizawa Y, Deguchi T, Ito M. Super pulse CO2 laser for bracket bonding and debonding. European Journal of Orthodontics. 1999; 21:193-198.

11. Eliades T, Gioka C, Eliades G, Makou M. Enamel surface roughness following debonding using two resin grinding methods. European Journal of Orthodontics. 2004; 26(3):333-338.

12. Ozer T, Basaran G, Kama JD. Surface roughness of the restored enamel after orthodontic treatment. Am J Orthod Dentofacial Orthop. 2010; $137(3): 368-374$

13. Proffit, W R, H W Fields, D M Sarver. Contemporary Orthodontic Appliances. In: Contemporary Orthodontics. 2007 ; ;.414-417.

14. Bennett, C G, Shen C, Waldron J M. The effects of debonding on enamel surface. J Clin Orthod. 1984; 18:330-334.

15. Pont H B, Özcan M, Bagis B, Ren Y. Loss of surface enamel after bracket debonding: An in-vivo and ex-vivo evaluation. Am J Orthod Dentofacial Orthop. 2010; 138, 387.e1-387.e9:

16. Øgaard B, W A Brantley, T Eliades (eds). Oral microbiolical changes, long term enamel alterations due to decalcification and carious prophylactic aspects. In: Orthodontic Materials: Scientific and Clinical Aspects. 2001; 124-139. Stuttgart, Germany, Thieme.

17. Zarrinnia K, Eid N M, Kehoe M J. The effect of different debonding techniques on the enamel surface: an in vitro qualitative study. Am J Orthod Dentofacial Orthop. 1995; 108:284-293.

18. Karan S, Toroglu M S. Porcelain refinishing with two different polishing systems after orthodontic debonding. The Angle Orthod. 2008; 78:947-953.

19. Ahrari F, Akbari M, Akbari J, Dabiri G. Enamel surface roughness after debonding of orthodontic bracket and various clean-up techniques. Journal of Dentistry, Tehran University of Medical Sciences, Tehran, Iran, 2013; 10(1):82-93.

20. Diedrich P. Enamel alterations from bracket bonding and debonding: a study with the scanning electron microscopy. Am $J$ Orthod Dentofacial Orthop. 1981; 79:500-522. 